\title{
Long interspersed nucleotide acid element-1 ORF-1 protein promotes proliferation and invasion of human colorectal cancer LoVo cells through enhancing ETS-1 activity
}

\author{
M.Y. Li ${ }^{1 *}$, M. Zhu ${ }^{1 *}$, F. Feng ${ }^{2 *}$, F.Y. Cai ${ }^{1}$, K.C. Fan ${ }^{1}$, H. Jiang ${ }^{1}$, \\ Z.Q. Wang ${ }^{1}$ and E.Q. Linghu ${ }^{1}$ \\ ${ }^{1}$ Department of Gastroenterology, Nan Lou Division, \\ Chinese PLA General Hospital, Beijing, China \\ ${ }^{2}$ Department of Pharmacy, General Hospital of Shenyang Military Command, \\ Shenyang, China \\ *These authors contributed equally to this study. \\ Corresponding authors: Z.Q. Wang / E.Q. Linghu \\ E-mail: zhiqiangwangcn@163.com / enqianglinghu@126.com
}

Genet. Mol. Res. 13 (3): 6981-6994 (2014)

Received April 26, 2013

Accepted September 20, 2013

Published April 14, 2014

DOI http://dx.doi.org/10.4238/2014.April.14.13

\begin{abstract}
The human proto-oncogene long interspersed nucleotide acid element-1 (LINE-1) open reading frame-1 protein (ORF-1p) is involved in the progress of several cancers. The transcription factor ETS-1 can mediate the transcription of some downstream genes that play specific roles in the regulation of cancerous cell invasion and metastasis. In this study, the effects of LINE-1 ORF-1p on ETS-1 activity and on the proliferation and invasion of human colorectal cancer LoVo cells were investigated. Results showed that the overexpression of LINE-1 ORF$1 \mathrm{p}$ enhanced the transcription of ETS-1 downstream genes and increased their protein levels, and downregulation of the LINE-1 ORF-1p level by small interfering RNA (siRNA) reduced the transcriptional activation of ETS-1. In addition, overexpression of LINE-1 ORF-1p promoted LoVo
\end{abstract}


cell proliferation and anchor-independent growth, and a knockdown of the LINE-1 protein level by siRNA reduced the proliferation and anchorindependent growth ability of LoVo cells. In vivo data revealed that LINE-1 ORF-1p overexpression increased LoVo tumor growth in nude mice, whereas the siRNA knockdown of endogenous LINE-1 ORF-1p expression decreased LoVo cell growth in nude mice. Therefore, LINE1 ORF-1p could promote LoVo cell proliferation and invasion both in vitro and in vivo, indicating that it might be a useful molecular target for the treatment of human colorectal cancer.

Key words: LINE-1 ORF-1p; ETS-1; Colorectal cancer LoVo cell; Transcriptional activation; Proliferation; Anchor-independent growth

\section{INTRODUCTION}

Human proto-oncogene long interspersed nucleotide acid element-1 (LINE-1) is a human retrotransposon gene. The expression of LINE-1 in normal tissues and cells is very low (Roman-Gomez et al., 2005; Goodier et al., 2007), but increases significantly and shows greater activity in most immortalized and malignant cells (Pornthanakasem et al., 2008). Activated LINE-1 can also promote a variety of tumor cell transformations (Gasior et al., 2006; Xing et al., 2009), and is closely related with the development of a variety of tumors, such as breast cancer, lung cancer, and others (Estécio et al., 2007). The LINE-1 gene consists of two open reading frame (ORFs), ORF-1 and ORF-2, which encode the proteins LINE-1 ORF-1p and LINE-1 ORF-2p, respectively (Roman-Gomez et al., 2005). Previous studies of LINE-1 ORF-2p has mainly concentrated on the obvious retrotransposon activation of LINE-1 (Gasior et al., 2006; Goodier et al., 2007; Pornthanakasem et al., 2008). However, the specific roles and detailed mechanisms of LINE-1 ORF-1p remain largely unknown. LINE-1 ORF-1p can bind with its own DNA and RNA, playing roles as a LINE-1 ORF-2p co-regulator (Gasior et al., 2006; Goodier et al., 2007; Pornthanakasem et al., 2008; Xing et al., 2009). LINE-1 ORF-1p is widely expressed in tumor tissues, and likely promotes cell carcinogenesis through affecting the structure of chromatin (Roman-Gomez et al., 2005; Martin, 2006; Estécio et al., 2007). Furthermore, our preliminary findings (Gao et al., 2010, 2011; Feng et al., 2012; Wang et al., 2012) have indicated that, by itself, LINE-1 ORF-1p could promote the proliferations and anchorage-independent growth of human colorectal cancer (HCC) cell lines such as HepG2, BEL-7402, and SMMC-7721, the liver source immortalized cell line LO2, the breast cancer cell line MCF-7, and the lung cancer cell lines A549 and H460. Results of our previous studies have also revealed that LINE-1 ORF-1p can promote the transcriptional activity of the estrogen receptor (ER) (Wang et al., 2012) and androgen receptor (AR) (Li MS and Zhu M, unpublished results); however, the regulating activity of LINE-1 ORF-1p in other transcription factors and nucleus receptors has not yet been reported.

Transcription factor ETS-1 is an important downstream response factor of receptor tyrosine c-Met (Saeki et al., 2002), and plays an important role in tumor development and invasion (Jiang et al., 2001). ETS-1 could mediate the transcription of a variety of invasion-related genes including MMP-1, MMP-9, u-PA, and c-Met, which are all downstream genes of ETS-1 (Bu et al., 2006). Previous studies have suggested that constitutive overexpression of the c-Met gene 
in a variety of tumor cells could increase the ETS-1 transcriptional activity. Activated ETS-1 further enhances the transcription of c-Met (Bu et al., 2006; Jin et al., 2011). Such a positive feedback regulation loop might lead to increased invasive abilities of tumor cells; therefore, blocking this mechanism might be an effective way to reduce tumor cell invasion and metastasis.

Colorectal cancer seriously harms human health, and the discovery and identification of colorectal cancer-related markers will be of great significance for identifying the specific molecular mechanism underlying the occurrence and development of this disease. HCC is characterized by high aggressive and metastasis activation, and research into the genes related to metastasis and invasion may have great significance for colorectal cancer treatment. The APC, DCC, MSH2, TP53, and RAS genes play specific roles in the progress of colorectal cancer (Rapozo et al., 2009). As an important transcription factor in tumor cell invasion and metastasis, ETS-1 shows certain correlations with the clinical staging, prognosis, and progress of colorectal cancer (Wai et al., 2006). Basic research has also shown that ETS-1 is involved in anti-apoptosis, proliferation, accelerated invasion, and metastasis regulation through promoting the transcription of downstream genes associated with invasion and Cyclin $\mathrm{D}$ expression (Cho et al., 2007; Peng et al., 2009). The activity of transcription factors is modulated by a large number of co-regulatory factors, including SRC-1, SRC-2, SMRT, and NcoR, among others (Harris et al., 2010). It is valuable to identify novel co-regulatory factors for the various activities of ETS-1. LINE-1 ORF-1p is a nucleoprotein that is generally highly expressed in tumor cells, but its biological function is not yet fully understood. Given the evidence that LINE-1 ORF-1p might influence chromatin structure (chromosome large-scale unfolding) (Pei et al., 2005) and modulate the transcriptional activity of ER (Wang et al., 2012), AR, PXR, LXR, Smad4, and CAR (Li MS, Zhu M and Wang ZQ, unpublished results), we hypothesize that LINE-1 ORF-1 p might also be involved in the regulation of colorectal cancer via modulating ETS-1 activity. We also found that LINE-1 ORF-1p could increase AR transcriptional activity through its direct interaction and impact on the migration of the nucleus and cytoplasm (Li MS, Zhu M and Wang ZQ, unpublished results). In this study, a small interfering RNA (siRNA) expression vector of LINE-1 ORF-1 and a LINE-1 ORF-1 expression vector were used. HCC LoVo cells were stably transfected with plasmids. A Western blot assay was used to verify the transfected cell lines. The effects of LINE-1 ORF-1p on ETS-1 activity and the proliferation and invasion of LoVo cells were investigated.

\section{MATERIAL AND METHODS}

\section{Main instruments and reagents}

The sodium dodecyl sulfate-polyacrylamide gel electrophoresis (SDS-PAGE) instrument was obtained from the Beijing Liuyi Instrument Factory (China), and the semi-transmembrane instrument and microplate reader were purchased from Bio-Rad (USA). The following reagents were used: Lipofectamine (Invitrogen Corp., USA), Dulbecco's modified Eagle's medium (DMEM; Hyclone Company, USA), fetal bovine serum (FBS; Gibco, USA), and neomycin G418 (Sigma Company, USA). The following antibodies were used: rabbit antiLINE-1 ORF-1p polyclonal antibodies (Cui et al., 2011), anti-GADPH polyclonal rabbit antibody (Sigma), horseradish peroxidase (HRP)-conjugated goat-anti-rabbit IgG (Santa Cruz, USA), rabbit anti-ETS-1 monoclonal antibody (Sigma), rabbit anti-MMP-1, anti-MMP-9 
polyclonal antibody, and anti-Survivin monoclonal antibodies (Santa Cruz). The following cells were used: colorectal cancer cell lines LoVo, HR8348, and HT29, the HCC cell line HepG2, the breast cancer cell line MCF-7, and the lung cancer cell line A549 (Chinese Academy of Medical Sciences, China). The plasmids LINE-1 ORF-1p, FLAG-LINE-1 ORF-1p expression vector and empty vector, LINE-1 ORF-1p siRNA expression vector, and the control siRNA vector were constructed according to previously published methods (Gao et al., 2010, 2011; Feng et al., 2012; Wang et al., 2012). The cDNA sequence of ETS-1 was cloned into a pIRES vector linked with FLAG at the amino terminus by polymerase chain reaction using the cDNA library (Invitrogen) as a template. The promoter region sequences of the luciferase reporter genes MMP-1-Luc, u-PA-Luc, c-Met-Luc, and MMP-9-Luc were synthesized using chemical synthesis methods (Gene Ray, USA). The promoter sequences were inserted directly into the pGL-4.26 vector (Promega, USA).

\section{Stable transfection of LoVo cells}

Plasmids were transfected into LoVo cells using Lipofectamine 2000. The transfected cells were cultured in $500 \mu \mathrm{g} / \mathrm{mL} \mathrm{G} 418$ for approximately 1-2 months. Then, individual clones were screened by Western blot using anti-FLAG monoclonal antibody or anti-LINE-1 ORF$1 \mathrm{p}$ antibody. Similar results were observed with stable transfection or transient transfection, individual clones, or pooled clones.

\section{Western blot assay}

LoVo cells were seeded and cultured on 6-well plates. Cells were then transfected with plasmids and harvested after $48 \mathrm{~h}$. Total protein samples were prepared by SDS-PAGE and transprinted to the nitrocellulose membrane, followed by blocking with $10 \%$ bovine serum albumin in Tris-buffered saline with Tween (TBST) buffer and incubation with antibodies. Then, membranes were incubated with HRP-conjugated secondary antibodies, and washed with TBST buffer 3 times. Finally, membranes were visualized. The blots were performed three times independently and yielded similar results. When incubating the HRP-FLAG monoclonal antibody, the blots were visualized without incubating with secondary antibody.

\section{Luciferase analysis}

Transfected LoVo cells were seeded on 24-well plates with DMEM supplemented with $10 \%$ charcoal-stripped FBS (Hyclone). Transfections were performed using Lipofectamine 2000. Cells were co-transfected with luciferase reporters and harvested for the luciferase and $\beta$-galactosidase activity analyses (Reid et al., 2003).

\section{Co-immunoprecipitation (IP) analysis}

LoVo cells were transfected with expression plasmids using Lipofectamine 2000. Cells were harvested and lysed in the IP buffer after being cultured for $24 \mathrm{~h}$. The co-IP analysis was performed with anti-FLAG monoclonal antibody and then detected by immunoblotting (IB; Reid et al., 2003). 


\section{Methylthiazol tetrazolium (MTT) analysis}

After transfection with appropriate plasmids, LoVo cells were inoculated on a 96-well plate, adding $100 \mathrm{~mL} 50 \mathrm{mM}$ MTT saturated solution at a fixed time every day. After incubation for $4 \mathrm{~h}$, the medium was disposed of, and $100 \mathrm{~mL} \mathrm{10 \%} \mathrm{SDS} \mathrm{solution} \mathrm{was} \mathrm{added.} \mathrm{After}$ mixing and oscillation, the optical density (OD) of the solution at $490 \mathrm{~nm}$ was detected in a microplate reader, and the growth curve was drawn. The OD at $490 \mathrm{~nm}$ reflected the relative number of viable cells.

\section{Soft agar colony analysis}

LoVo cells were stably transfected with plasmids. Then, 500 cells per well were plated on a 6-well plate, with a bottom layer of $0.7 \%$ low melting temperature agar in DMEM and a top layer of $0.25 \%$ agar in DMEM. Colonies were scored after 3-4 weeks of growth (Reid et al., 2003; Chen et al., 2005; Hu et al., 2010; Zhang et al., 2012).

\section{Nude mice subcutaneous tumor experiments}

A total of $5 \times 10^{5}$ LoVo cells were seeded in the right rear of 5-week-old male BALB/c nude mice $(\mathrm{N}=5)$, and the exudate at the inoculation site, tumor growth, and nude mice condition were observed. The maximum $(\mathrm{L}, \mathrm{cm})$ and minimum $(\mathrm{D}, \mathrm{cm})$ tumor diameters were measured, and the tumor volume was calculated as follows: tumor volume $\left(\mathrm{cm}^{3}\right)=\mathrm{L}^{2} \times \mathrm{D} \times 1 / 2$. Approximately 10 weeks after cell transplantation, all mice were sacrificed. The tumor mass was dissected, and the tumor mass and nude mice mass were determined. Tumor mass weight was used for calculations of relative multiples: (tumor mass weight of experimental group / nude mice body weight of experimental group) / (tumor mass weight of control group / nude mice body weight of control group). The tumor mass was rinsed with ice-cold $0.9 \%$ saline (with the addition of 1:500 protease inhibitor), and then placed in a 5-mL centrifuge tube. Immediately after, $1 \mathrm{~mL}$ lysis buffer (pre-cooled to $0^{\circ} \mathrm{C}$ ) (with 1:500 protease inhibitor) was added, followed by homogenization in an ice bath. Western blot analysis was used to analyze the protein expressions of Survivin, MMP-1, and MMP9 in the tumor mass.

\section{Statistical analysis}

Statistical analysis was performed using the SPSS 13.0 software. Measurement data are reported as means \pm standard deviation, and the difference between the two groups was analyzed using the least squares difference (LSD) Student $t$-test; $\mathrm{P}<0.05$ reflected a statistically significant difference.

\section{RESULTS}

\section{ETS-1 and LINE-1 ORF-1p expression spectra in different tumor cell lines}

As shown in Figure 1, LINE-1 ORF-1p and ETS-1 were expressed over a wide range of cell types, such as the colon cancer cell lines LoVo, HR8348, and HT29, the HCC cell line 
HepG2, the breast cancer cell line MCF-7, and the lung cancer cell line A549. ETS-1 was highly expressed in LoVo and HepG2 cells, whereas the expression level of LINE-1 ORF-1p was relatively lower only in the HT29 colorectal cancer cell line and the breast cancer cell line MCF-7. This indicated that ETS-1 was mainly present in highly invasive colorectal cancer cell lines (the LoVo malignant degree was the highest) and in tumors with poor prognosis (HepG2 cell lines), whereas LINE-1 ORF-1p was widely expressed in all tumor cell lines. ETS-1 and LINE-1 ORF-1p showed higher expression levels in LoVo cells; therefore, LoVo was selected as the colorectal cancer model in our study.

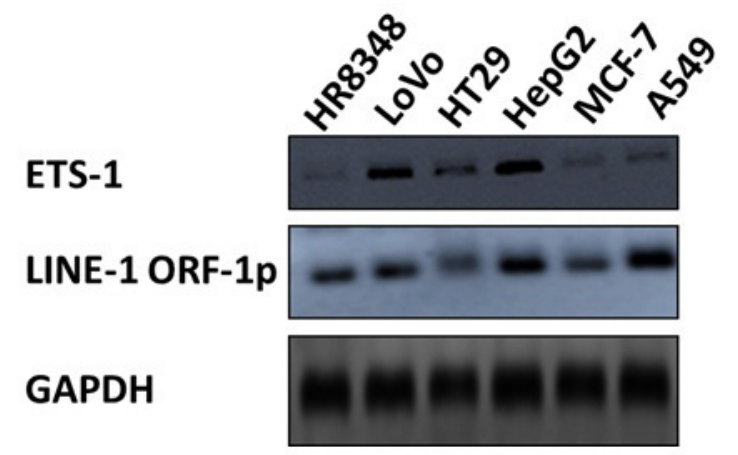

Figure 1. Expression spectrum of LINE-1 ORF-1p and ETS-1 in cancerous cells. Proteins extracted from the HR8348, LoVo, HT29, HepG2, MCF-7, and A549 cell lines were analyzed by Western blot with anti-GAPDH, anti-ETS-1, anti-LINE-1 ORF-1p.

\section{Modulation of LINE-1 ORF-1p on the transcriptional activity of ETS-1}

Luciferase assays were performed to examine whether or not LINE-1 ORF1-p could modulate the transcriptional activity of ETS-1 in LoVo cells. Results showed that compared with the empty vector, overexpression of LINE-1 ORF-1p in stably transfected LoVo cell lines could obviously increase the activity of MMP-1-Luc (Figure 2A), MMP-9-Luc (Figure 2B), uPA-Luc (Figure 2C), and c-Met-Luc (Figure 2D). Furthermore, compared with control siRNA, LINE-1 ORF-1p siRNA could significantly reduce the activities of reporter genes of MMP-1Luc (Figure 2A), MMP-9-Luc (Figure 2B), u-PA-Luc (Figure 2C), and c-Met-Luc (Figure 2D). These results suggest that LINE-1 ORF-1p could elevate the transcriptional activity of ETS-1.

\section{Interaction of LINE-1 ORF-1p with ETS-1 in LoVo cells}

Interactions between LINE-1 ORF-1p and ETS-1 in LoVo cells were investigated in the IP assay. LoVo cells were transfected with the FLAG-LINE-1 ORF-1p expression vector, FLAG-ETS-1 expression vector, or FLAG empty vector. Cells were harvested, the IP assay was performed, and each antibody was detected through IB assays. The co-IP results showed that LINE-1 ORF-1p interacted with LoVo cells' endogenous ETS-1 (Figure 3A) (IP: FLAG; IB: ETS-1; IP: FLAG; IB: FLAG). The FLAG empty vector could not interact with LoVo cells' endogenous ETS-1 (Figure 3A) (IP: FLAG; IB: ETS-1; IP: FLAG; IB: FLAG). In addition, the reverse IP experimental results showed that FLAG-ETS-1 could interact 
with LoVo cells' endogenous LINE-1 ORF-1p (Figure 3B) (IP: FLAG; IB: LINE-1 ORF-1p; IP: FLAG; IB: FLAG), whereas the FLAG empty vector could not interact with LoVo cells' endogenous LINE-1 ORF-1p (Figure 3A) (IP: FLAG; IB: LINE-1 ORF-1p; IP: FLAG; IB: FLAG). Therefore, LINE-1 ORF-1p appears to physically interact with ETS-1 in LoVo cells.

A

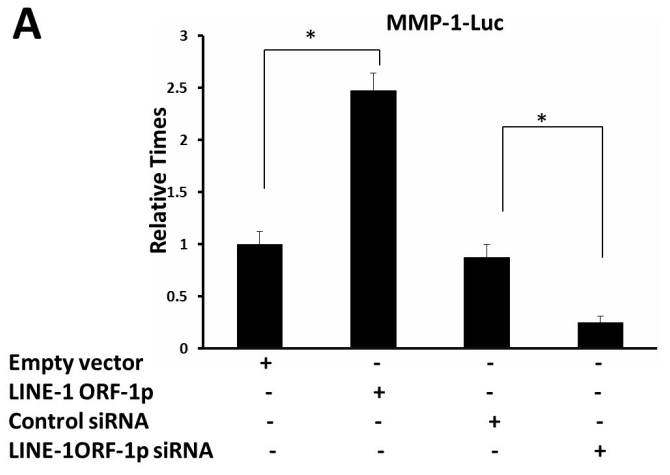

C

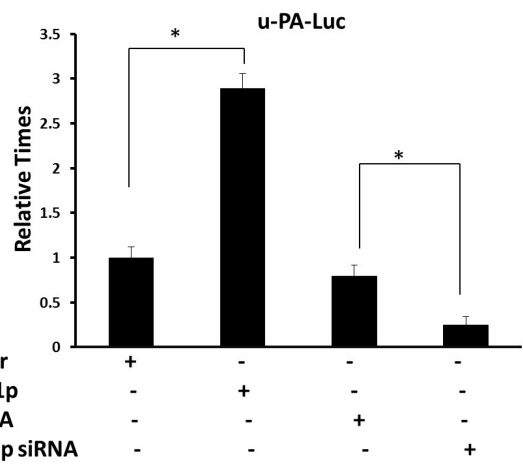

B

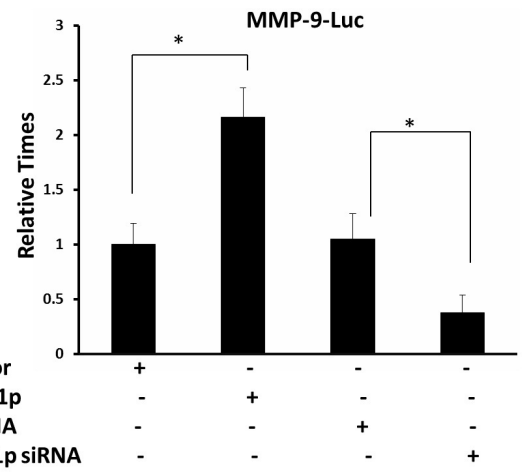

D
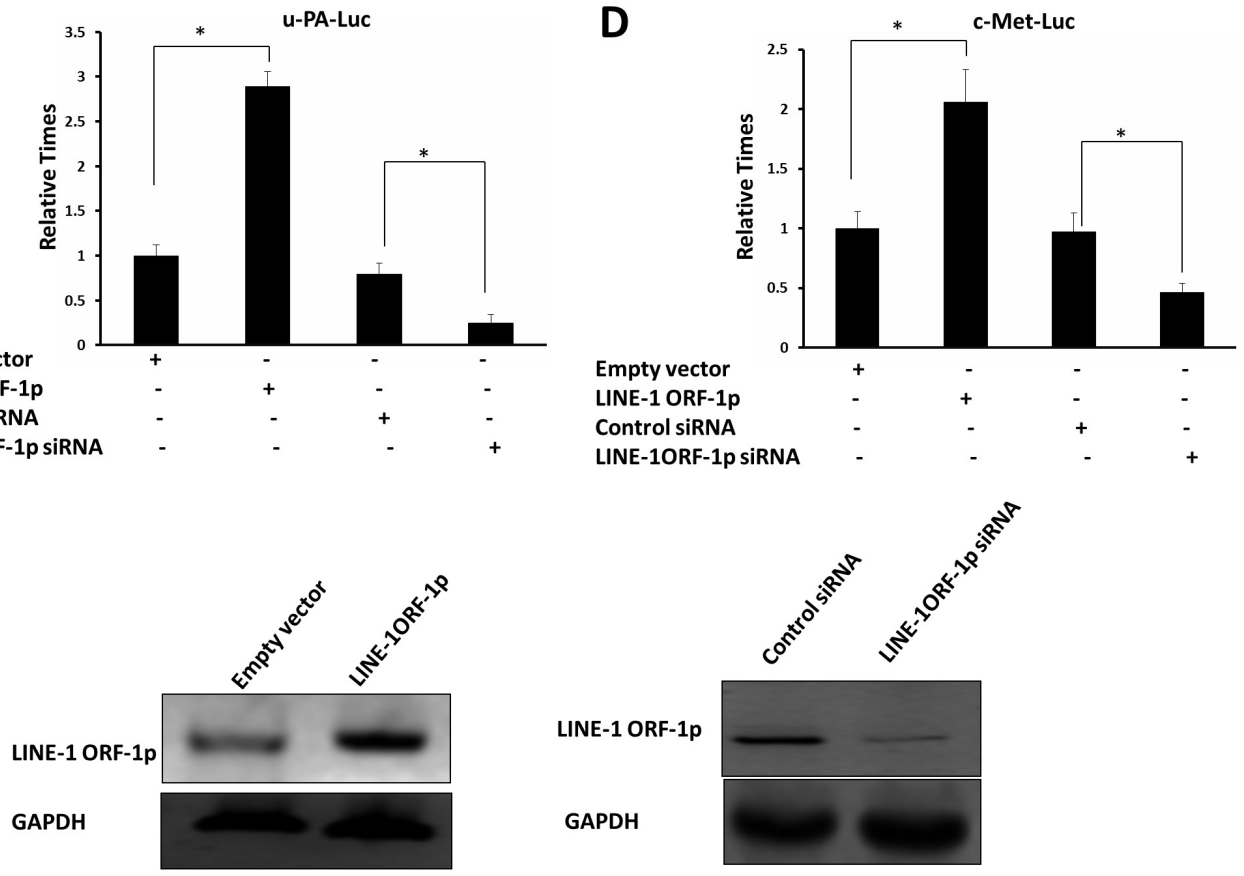

Figure 2. LINE-1 ORF-1p enhanced the transcriptional activity of ETS-1. LoVo cells stably transfected with empty vector or LINE-1 ORF-1p expression vector, control siRNA empty vector or LINE-1 ORF-1p siRNA expression vector. A. LoVo cells co-transfected with MMP-1-Luc reporter gene; B. LoVo cells co-transfected with MMP-9Luc reporter gene; C. LoVo cells co-transfected with u-PA-Luc reporter gene; D. LoVo cells co-transfected with c-Met-Luc reporter gene. $* \mathrm{P}<0.05$. 


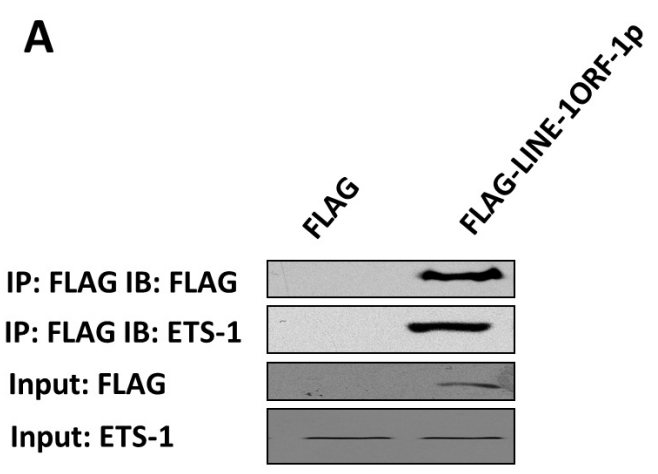

B

IP: FLAG IB: FLAG

IP: FLAG

IB: LINE-1 ORF-1p Input: FLAG

Input: LINE-1 ORF-1p

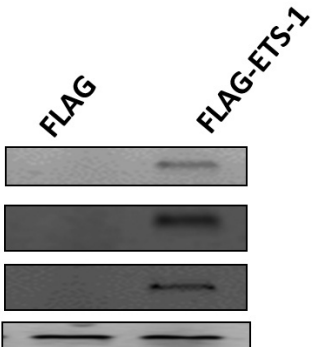

$\longrightarrow$

Figure 3. LINE-1 ORF-1p physically interacted with ETS-1 in LoVo cells. A. LoVo cells transfected with FLAG empty vector, FLAG-LINE-1 ORF-1p expression vector. B. LoVo cells transfected with FLAG-ETS-1 expression vector or FLAG empty vector. The immunoprecipitation (IP) analysis was performed with anti-FLAG antibody, and the immunoblotting (IB) analysis was performed with anti-FLAG antibody, anti-LINE-1 ORF-1p antibody or anti-ETS-1 antibody.

\section{Effect of LINE-1 ORF-1p on LoVo cell proliferation}

An MTT assay was performed to determine whether LINE-1 ORF-1p might affect the proliferation of colorectal cancer cells. LoVo cells were stably transfected with the LINE-1 ORF-1p expression vector, empty vector, or with the LINE-1 ORF-1p siRNA vector or siRNA empty vector, and then MTT assays were performed. As shown in Figure 4, overexpression of LINE-1 ORF-1p could significantly promote LoVo cell proliferation (Figure 4A), which started showing a significant difference compared to the empty vector as of the 4th day $(\mathrm{P}<0.05)$. LINE-1 ORF-1p siRNA could significantly inhibit the growth of LoVo cells (Figure 4B), showing a significant difference compared to the control siRNA empty vector as of the 3rd day ( $\mathrm{P}$ $<0.05)$. These results suggested that LINE-1 ORF-1p could promote LoVo cell proliferation.
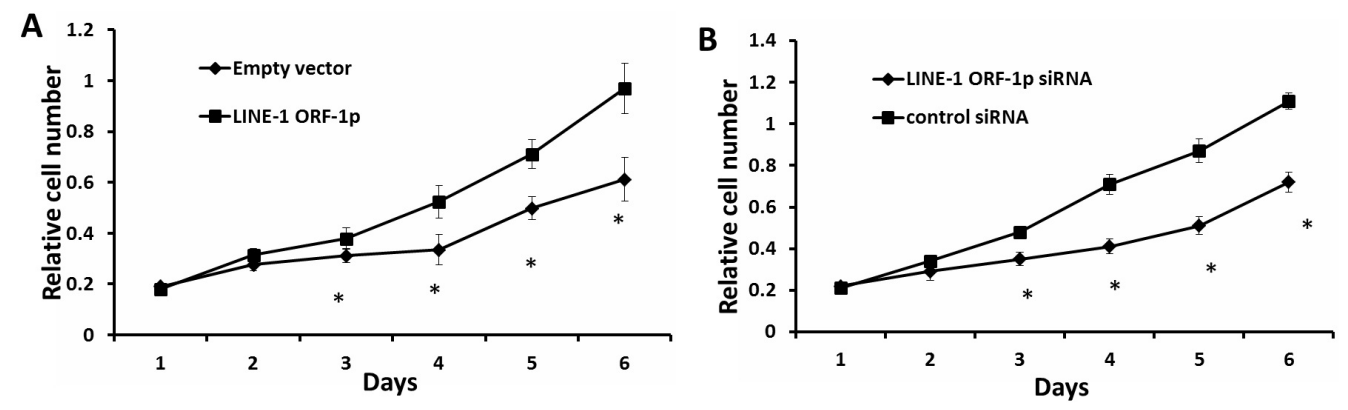

Figure 4. LINE-1 ORF-1p promoted LoVo cell growth. A. LoVo cells stably transfected with LINE-1 ORF-1p vector or the empty vector; B. LoVo cells stably transfected with LINE-1 ORF-1p siRNA vector or the control siRNA vector. Then, cell numbers were determined by the MTT assay. ${ }^{*} \mathrm{P}<0.05$ vs empty vector or LINE-1 ORF$1 \mathrm{p}$ vector $(\mathbf{A}) ; * \mathrm{P}<0.05$ vs LINE-1 ORF-1p siRNA vector or control siRNA vector (B). 


\section{Effect of LINE-1 ORF-1p on the anchorage-independent growth ability of LoVo cells}

To corroborate the results of the MTT assay, it was necessary to determine the effects of LINE-1 ORF-1p on anchor-independent growth; therefore, a soft agarose assay was performed. As shown in Figure 5, overexpression of LINE-1 ORF-1p could significantly increase the LoVo colony size (Figure 5A) and the number of colonies formed (Figure 5B). The siRNA targeting LINE-1 ORF-1P significantly reduced the LoVo colony size (Figure $5 \mathrm{~A}$ ) and the colony number (Figure 5B) compared to the control siRNA empty vector ( $\mathrm{P}$ $<0.05)$. This suggested that LINE-1 ORF-1p could enhance the anchorage-independent growth ability of LoVo cells, and play potential roles in promoting LoVo cell migration and invasion.
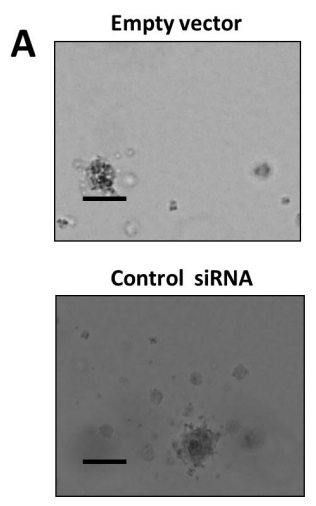
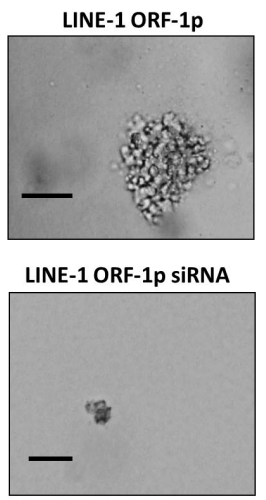

B

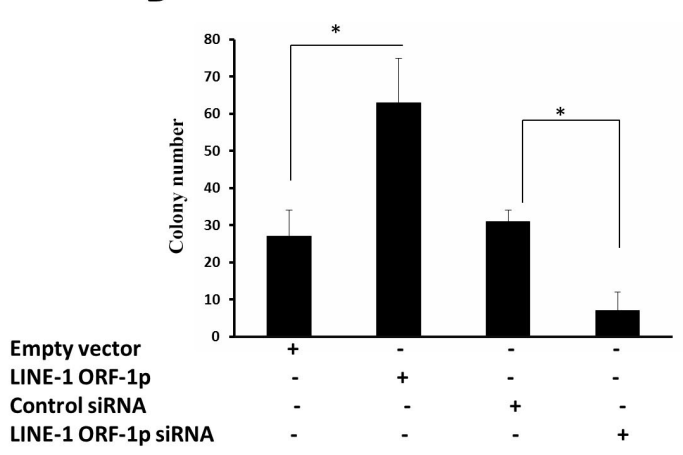

Figure 5. LINE-1 ORF-1p enhanced LoVo cells anchor-independent proliferation. A. LoVo cells stably transfected with LINE-1 ORF-1p vector, empty vector, LINE-1 ORF-1p siRNA vector and control siRNA vector seeded on soft agar and assayed for colony number after 3 weeks. B. Colony numbers (means \pm SD) of triplicate independent experiments. ${ }^{*} \mathrm{P}<0.05$ vs FLAG or FLAG-LINE-1 ORF-1p, ${ }^{*} \mathrm{P}<0.05$ vs LINE-1 ORF-1p siRNA vector or control siRNA vector.

\section{Effect of LINE-1 ORF-1p on in vivo LoVo cell growth}

To confirm the results of the MTT and soft agarose assays, the in vivo regulatory roles of LINE-1 ORF-1p in LoVo cells were evaluated. Results showed that LINE-1 ORF$1 \mathrm{p}$ enhanced the tumorigenic ability of LoVo cells in nude mice. The LoVo cell tumor mass caused by LINE-1 ORF-1p overexpression was significantly greater than that of the control (Figure 6A), and the growth rate of the tumor mass was accelerated (Figure 6B), showing a significant difference compared to the empty vector as of the 6th week $(\mathrm{P}<0.05$; Figure 6B). Conversely, LINE-1 ORF-1p siRNA reduced the tumor volume (Figure 6A and $\mathrm{C}$ ), which started showing a significant difference compared to the control siRNA empty vector as of the 7 th week $(\mathrm{P}<0.05)$ (Figure $6 \mathrm{C})$. The tumor mass results also showed that LINE-1 ORF-1p could promote LoVo cell tumor formation, whereas reducing the expression of LINE-1 ORF-1p could retard the formation of the tumor mass. 
Therefore, LINE-1 ORF-1p could promote LoVo cells in nude mice to form tumors, and likely plays a role in promoting the metastasis and invasion of tumor cells in the body.
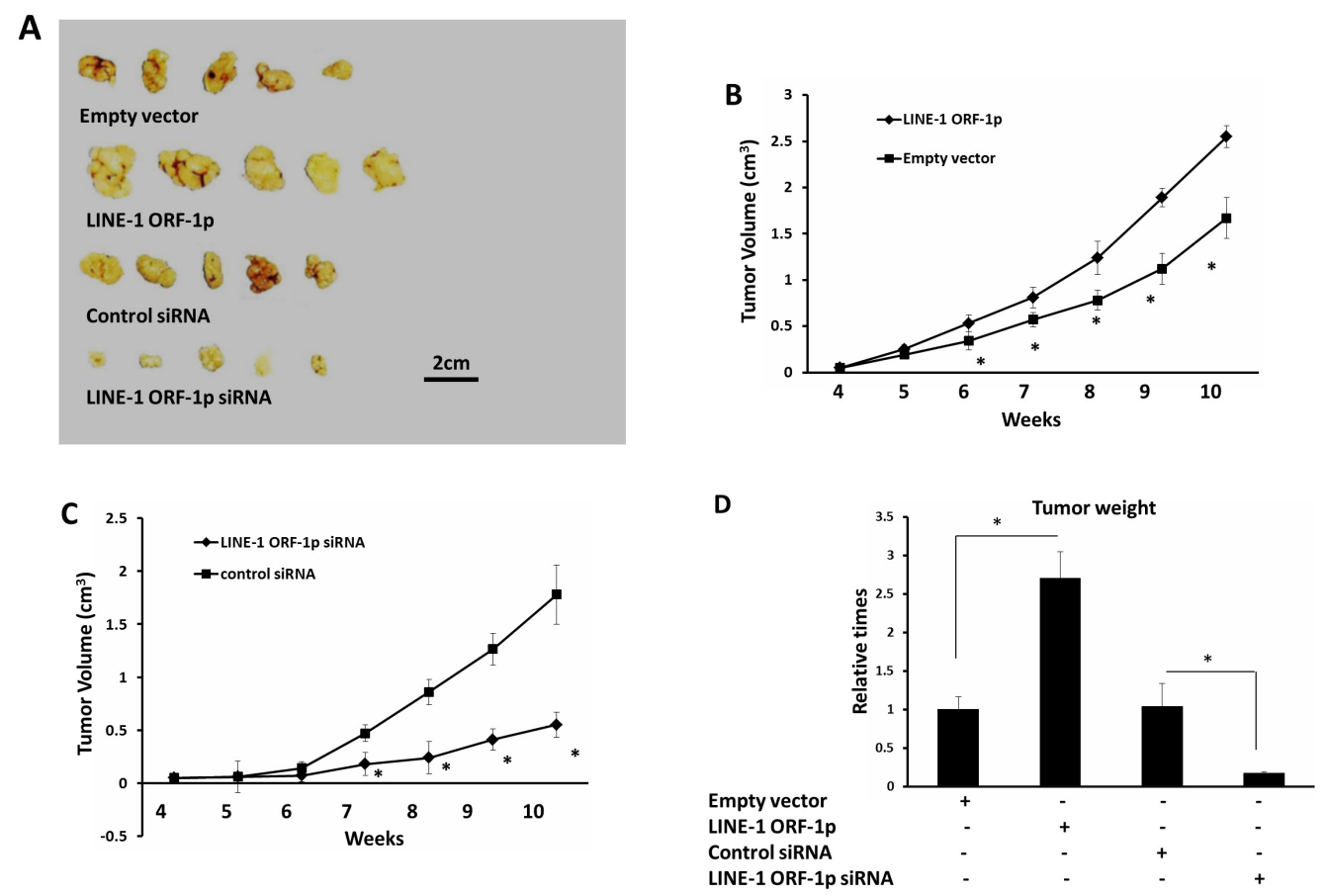

Figure 6. LINE-1 ORF-1p could promote LoVo cells tumorigenic ability. LoVo cells were stably transfected with FLAG-LINE-1 ORF-1p vector or FLAG empty vector, or LINE-1 ORF-1p siRNA vector or control siRNA vector. Cells were injected into nude mice. At the indicated times, tumors were measured with tumor volume (A-C) or tumor weight (D) (means $\pm \mathrm{SD} ; \mathrm{N}=5) .{ }^{*} \mathrm{P}<0.05$ vs FLAG or FLAG-LINE-1 ORF-1p (B), ${ }^{*} \mathrm{P}<0.05$ vs LINE-1 ORF-1p siRNA vector or control siRNA vector $(\mathbf{C})$.

\section{Western blot assays}

Western blot assays were performed to corroborate the results of the luciferase assays. To determine whether LINE-1 ORF-1 affected the protein expression of ETS-1 downstream genes, total proteins were extracted from the tumor mass, and the expressions of LINE-ORF-1p, MMP-1, MMP-9, and Survivin were detected, using GAPDH as the internal reference. Results (Figure 7A and B) indicated that overexpression of LINE-1 ORF-1 $p$ increased the MMP-1, MMP-9, and Survivin expressions, whereas downregulation of the LINE-1 ORF-1p protein level through siRNA reduced the expressions of MMP-1, MMP-9, and Survivin. This indicated that LINE-1 ORF-1p could increase the protein expressions of ETS-1 downstream genes and Survivin, which are all important for cell survival/anti-apoptosis. 
A

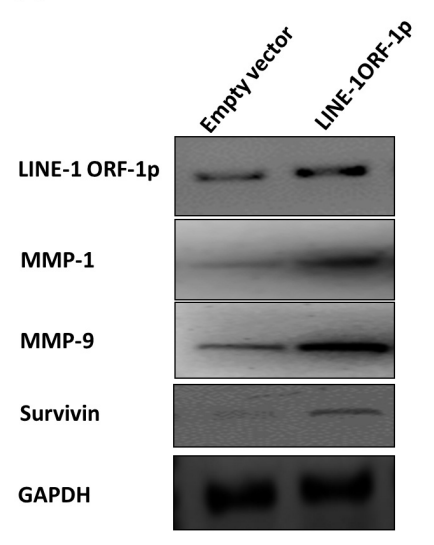

B

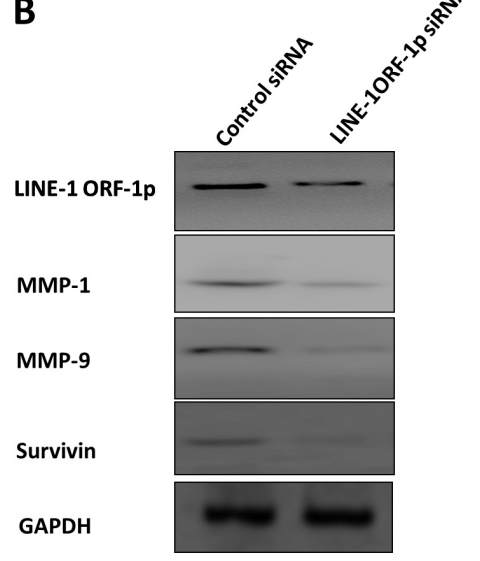

Figure 7. LINE-1 ORF-1p modulated MMP-1, MMP-9, and Survivin protein expression. Tumor tissues were detected by the anti-LINE-1 ORF-1p, anti-MMP-1, anti-MMP-9, anti-Survivin and anti-GAPDH antibodies. A. LoVo cells stably transected with FLAG vector or FLAG-LINE-1 ORF-1p vector; B. LoVo cells stably transected with control siRNA vector or LINE-1 ORF-1p siRNA vector.

\section{DISCUSSION}

In the present study, LINE-1 ORF-1p enhanced ETS-1 transcriptional activity through physical interactions between the proteins. Overexpression of LINE-1 ORF-1p increased the transcription and protein levels of the ETS-1 downstream genes MMP-1, MMP-9, u-PA, and c-Met. In addition, LINE-1 ORF-1p promoted the proliferation of LoVo cells in vitro and in vivo. Anchorage-independent growth is the basis of tumor cell migration and invasion; however, in in vivo experiments, nude mouse subcutaneous tumor formation provides direct evidence of tumor cell invasion and malignant transformation. These results indicated that LINE-1 ORF-1p plays roles in the metastasis and invasion of LoVo cells.

This study also found that LINE-1 ORF-1p could elevate the expression of Survivin, which is known to be the most powerful anti-apoptotic gene. Therefore, corroborating the results of our previous study, LINE-1 ORF-1p was shown to be mainly involved in the regulation of some important transcription factors, cell proliferation, cell cycle progression, and cell apoptosis (Gao et al., 2011; Feng et al., 2012; Wang et al., 2012). These roles are significantly different from the retrotransposition mechanisms of LINE-1 ORF-2p, which might result in a large number of mutations, and ultimately lead to instability in the genetic material and cellular transformations. On the other hand, in the last 20 years, studies of LINE-1 ORF-1p have focused on retrotransposition because LINE-1 ORF-1p was shown to be the regulatory factor of LINE-1 ORF-2p. Recently, however, it was reported that LINE-1 ORF-1p was also likely to play its own role in tumor development. LINE-1 ORF-1p was highly expressed in a variety of tumor tissues associated with poor prognosis (Cho et al., 2007; Harris et al., 2010), but the specific molecular mechanism of its role was still not entirely clear. The results of this study suggested that LINE-1 ORF-1p is not only the main adjustment factor of LINE-1 ORF-2p, but also plays specific roles itself. 
Although some evidence indicated that LINE-1 ORF-1p played roles in the development of cancer cell proliferation, the detailed mechanisms were still not fully understood. Therefore, studies investigating the function of LINE-1 ORF-1p are valuable to elucidate the development mechanism of malignant tumors. LINE-1 ORF-1p has been recognized in the liver tumor cell lines Bel-7402, SMMC-7721, and HepG2, and in liver-sourced immortalized cell lines, such as LO2. It can promote the cell cycle progress, and protect cells against apoptosis in the lung cancer cell lines H460 and A549 and in the gastric cancer cell line BGC823 (Gao et al., 2010, 2011; Feng et al., 2012; Wang et al., 2012). Results of this study confirmed that LINE-1 ORF-1p also plays important roles in the proliferation and invasion of colorectal cancer. Combined with this study, we systematically established a series of tumor cell models covering the function of LINE-1 ORF-1p in several major tumor types, thus providing a solid foundation for future research of LINE-1 ORF-1p.

As a transcription factor, ETS-1 has a DNA binding domain and a transcriptional activation domain. Like other transcription factors (ER, AR, p53, and Smad4), ETS-1 also plays its own roles in subcellular localization, transcriptional activation, and degradation, and a large number of co-regulators are involved in regulating ETS-1 functions (Reid et al., 2003). The results of this study suggested that LINE-1 ORF-1p might act as an activation co-regulator/co-activator of ETS-1. This was consistent with our previous studies, and established a clear molecular mechanism for previous results. The mechanisms of co-regulation of the transcription factor were evident in four aspects (Choi et al., 2009; Ding et al., 2009; Zhang et al., 2012): a) physical interaction, b) promoting the recruitment of transcription factor with their DNA binding elements, c) modulating the cytoplasmic/nucleus translocation of the transcription factor, and d) recruiting other co-regulators to the promoter of the downstream gene. This study demonstrated that LINE-1 ORF-1p increased ETS-1 transcriptional activity through physical interactions, but whether LINE-1 ORF-1p might function through other mechanisms remains unknown. Nevertheless, future studies of the molecular mechanism of LINE-1 ORF$1 \mathrm{p}$ in adjusting important transcription factors could be facilitated based on this study.

Molecular mechanisms of colorectal cancer are complex, and the events follow an obvious chronological sequence such as the mutations of corresponding oncogenes and tumor suppressor genes. Mutations of the APC and MSH2 genes occur mainly during the initial stage, and abnormal activation of KRAS occurs in the early stages of adenoma (Rapozo et al., 2009). DCC and P53 play a role in the late stages of adenoma, while there is no clear target molecule in the adenocarcinoma stage or in the recurrence, metastasis, or invasion stages of colorectal cancer (Rapozo et al., 2009). HCC is characterized by highly aggressive and metastatic activation; therefore, it would be of great significance to identify new therapy target molecules and clinical indicators of colorectal cancer. The purpose of this study was to initially examine the function and mechanism of potential target molecules, LINE-1 ORF-1p and ETS-1, in the migration and invasion of colorectal cancer. The c-Met/ETS-1 signaling pathway plays a central role in tumor cell migration and invasion, and the proto-oncogene LINE-1 ORF-1p possibly functions through the ETS-1, thus promoting the proliferation and invasion of colon cancer cells. This study not only found that ETS-1 might act as a protein factor in the development and invasion of colorectal cancer, but also expanded the scope of LINE-1 ORF-1p, demonstrating its possible mechanism of action. We intend to conduct further studies on the significance and detailed molecular mechanisms of the interaction between LINE-1 ORF-1p and ETS- 1 in the development of colorectal cancer. 
In conclusion, this study established a method for investigating the interaction between LINE-1 ORF-1p and ETS-1 in a colorectal cancer cell line LoVo, and identified its novel roles in the proliferation and invasion of colorectal cancer.

\section{REFERENCES}

Bu S, Yamanaka M, Pei H, Bielawska A, et al. (2006). Dihydrosphingosine 1-phosphate stimulates MMP1 gene expression via activation of ERK1/2-Ets1 pathway in human fibroblasts. FASEB J. 20: 184-186.

Chen G, Nomura M, Morinaga H, Matsubara E, et al. (2005). Modulation of androgen receptor transactivation by FoxH1. A newly identified androgen receptor corepressor. J. Biol. Chem. 280: 36355-36363.

Cho NY, Kim BH, Choi M, Yoo EJ, et al. (2007). Hypermethylation of CpG island loci and hypomethylation of LINE-1 and Alu repeats in prostate adenocarcinoma and their relationship to clinicopathological features. J. Pathol. 211: 269-277.

Choi WI, Jeon BN, Yun CO, Kim PH, et al. (2009). Proto-oncogene FBI-1 represses transcription of p21CIP1 by inhibition of transcription activation by $\mathrm{p} 53$ and Sp1. J. Biol. Chem. 284: 12633-12644.

Cui J, Yang Y, Zhang C, Hu P, et al. (2011). FBI-1 functions as a novel AR co-repressor in prostate cancer cells. Cell Mol. Life Sci. 68: 1091-1103.

Ding L, Wang Z, Yan J, Yang X, et al. (2009). Human four-and-a-half LIM family members suppress tumor cell growth through a TGF-beta-like signaling pathway. J. Clin. Invest. 119: 349-361.

Estécio MR, Gharibyan V, Shen L, Ibrahim AE, et al. (2007). LINE-1 hypomethylation in cancer is highly variable and inversely correlated with microsatellite instability. PLoS One 2: e399.

Feng F, Gao XD, Lu YY, Zhang F, et al. (2012). Regulation of LINE-1 ORF-1p on hepatocellular carcinoma cells and immortalized hepatocellular cells. Med. J. Chin. PLA 37: 123-127.

Gao XD, Hu MM and Zhu YF (2010). The down-regulation of LINE-1 encoded ORF-1p impacts the characters of A549 cells. Zhongguo Shengwu Kexue Zazhi 30: 14-20.

Gao XD, Lu YY, Feng F and Wang CP (2011). Effects of LINE-1 ORF-1p overexpression on proliferation and anchorindependent growth of SMMC7721 hepatoma cell line. J. Clin. Hepatol. 14: 323-326.

Gasior SL, Wakeman TP, Xu B and Deininger PL (2006). The human LINE-1 retrotransposon creates DNA double-strand breaks. J. Mol. Biol. 357: 1383-1393.

Goodier JL, Zhang L, Vetter MR and Kazazian-Jr HH (2007). LINE-1 ORF1 protein localizes in stress granules with other RNA-binding proteins, including components of RNA interference RNA-induced silencing complex. Mol. Cell Biol. 27: 6469-6483.

Harris CR, Normart R, Yang Q, Stevenson E, et al. (2010). Association of nuclear localization of a long interspersed nuclear element-1 protein in breast tumors with poor prognostic outcomes. Genes Cancer 1: 115-124.

Hu MM, Zhu YF, Wang Y, Wang Y, et al. (2010). Prokaryotic expression of LINE-1 encoded protein L1 ORF-1p and preparation of anti-L1 ORF-1p antibody. China Biotechnol. 30: 7-10.

Jiang Y, Xu W, Lu J, He F, et al. (2001). Invasiveness of hepatocellular carcinoma cell lines: contribution of hepatocyte growth factor, c-met, and transcription factor Ets-1. Biochem. Biophys. Res. Commun. 286: 1123-1130.

Jin BF, Xia Q and Zhang XM (2011). Progress of AMMS in tumor research. Sci. China: Life Sci. 41: 807-811.

Martin SL (2006). The ORF1 protein encoded by LINE-1: structure and function during L1 retrotransposition. J. Biomed. Biotechnol. 2006: 45621.

Pei H, Li C, Adereth Y, Hsu T, et al. (2005). Caspase-1 is a direct target gene of ETS1 and plays a role in ETS1-induced apoptosis. Cancer Res. 65: 7205-7213.

Peng Y, Li H, Wu M, Wang X, et al. (2009). NGX6 inhibits AP-1 and Ets-1 expression and down-regulates cyclin D1 in human colorectal cancer. Acta Biochim. Biophys. Sin. 41: 504-514.

Pornthanakasem W, Kongruttanachok N, Phuangphairoj C, Suyarnsestakorn C, et al. (2008). LINE-1 methylation status of endogenous DNA double-strand breaks. Nucleic Acids Res. 36: 3667-3675.

Rapozo DC, Grinmann AB, Carvalho AT, de Souza HS, et al. (2009). Analysis of mutations in TP53, APC, K-ras, and DCC genes in the non-dysplastic mucosa of patients with inflammatory bowel disease. Int. J. Colorectal Dis. 24: 1141-1148.

Reid G, Hubner MR, Metivier R, Brand H, et al. (2003). Cyclic, proteasome-mediated turnover of unliganded and liganded ERalpha on responsive promoters is an integral feature of estrogen signaling. Mol. Cell 11: 695-707.

Roman-Gomez J, Jimenez-Velasco A, Agirre X, Cervantes F, et al. (2005). Promoter hypomethylation of the LINE-1 retrotransposable elements activates sense/antisense transcription and marks the progression of chronic myeloid 
leukemia. Oncogene 24: 7213-7223.

Saeki H, Oda S, Kawaguchi H, Ohno S, et al. (2002). Concurrent overexpression of Ets-1 and c-Met correlates with a phenotype of high cellular motility in human esophageal cancer. Int. J. Cancer 98: 8-13.

Wai PY, Mi Z, Gao C, Guo H, et al. (2006). Ets-1 and runx2 regulate transcription of a metastatic gene, osteopontin, in murine colorectal cancer cells. J. Biol. Chem. 281: 18973-18982.

Wang B, Feng F, Zhang F, Liu MY, et al. (2012). In vitro effect of long interspersed nucleotide acids element-1 ORF-1p on estrogen receptor $\alpha$ of positive human breast cancer cells. J. Chin. PLA Postgrad. Med. Sch. 33: 763-766.

Xing J, Zhang Y, Han K, Salem AH, et al. (2009). Mobile elements create structural variation: analysis of a complete human genome. Genome Res. 19: 1516-1526.

Zhang F, Feng F, Yang P, Li Z, et al. (2012). Four-and-a-half-LIM protein 1 down-regulates estrogen receptor alpha activity through repression of AKT phosphorylation in human breast cancer cell. Int. J. Biochem. Cell Biol. 44: 320-326. 